\title{
PRODUÇÃO DE PELOTAS AUTO-REDUTORAS A PARTIR DE LIXO ORGÂNICO DOMÉSTICO*
}

\author{
Alberto Eloy Anduze Nogueira ${ }^{1}$ \\ Cyro Takanor ${ }^{2}$ \\ Marcelo Breda Mourão ${ }^{3}$ \\ Adolfo Pillihuaman Zambrano ${ }^{4}$
}

\section{Resumo}

O lixo orgânico doméstico gera um problema de eliminação crescente, exigindo sistemas de processamento dispendiosos. É necessário encontrar novas aplicações para estes resíduos; uma poderia ser como matéria prima metalúrgica. Neste trabalho é estudado o desenvolvimento de pelotas auto-redutoras usando a pirólise de resíduos orgânicos, onde é gerado carbono e voláteis condensáveis e não condensáveis. $\mathrm{O}$ alcatrão resultante foi misturado com minério de ferro, pó de carvão e fluxante $(\mathrm{CaO})$, para produzir as pelotas. O carvão fluidizado cria uma forte aderência dos elementos, mostrando uma boa viabilidade do presente processo.

Palavras-chave: Auto-redução; Resíduos orgânicos; Pirólise; Alcatrão.

\section{THE SELF-REDUCING PELLET PRODUCTION FROM ORGANIC HOUSEHOLD WASTE}

\section{Abstract}

The domestic organic waste disposal generates an increasing problem requiring expensive processing systems. It is necessary to find new applications for these wastes; one could be as metallurgical raw material. This paper examines the development of self-reducing pellets using pyrolysis of organic waste, which generated carbon and condensable and non-condensable volatile. The resulting tar was mixed with iron ore, coal powder and flux $(\mathrm{CaO})$ to produce pellets. The fluidized coal creates a strong grip of the elements, showing a good feasibility of this process.

Keywords: Self-reduction; Organic waste; Pyrolysis; Tar.

1 PhD em engenharia Metalúrgica e de Materiais, Pesquisador, Escola de Metalurgia e Materiais da Universidade de São Paulo, São Paulo, SP, Brasil.

2 PhD em engenharia Metalúrgica e de Materiais, Professor Associado, Escola de Metalurgia e Materiais da Universidade de São Paulo, São Paulo, SP, Brasil.

3 PhD em engenharia Metalúrgica e de Materiais, Professor Associado, Escola de Metalurgia e Materiais da Universidade de São Paulo, São Paulo, SP, Brasil.

4 PhD em engenharia Metalúrgica e de Materiais, Professor Associado, Pontificia Universidad Católica del Perú, Lima, Perú. 


\section{INTRODUÇÃO}

A eliminação de resíduos é um problema crescente. Além de ser um problema de saúde pública o recolhimento de lixo, o processamento e transporte em grandes distâncias aumenta os custos [1]. Existem várias técnicas para lidar com o lixo. Existe a reciclagem, os aterros sanitários, a compostagem e a incineração. Reciclagem tem limitações, como a quantidade e a utilização que se pode dar aos polímeros [2]. As novas regras aplicáveis aos aterros exigem que o material esteja limpo e estabilizado, forçando o pré-tratamento de resíduos. Os aterros têm um controle rigoroso, levando em consideração a infiltração de chorume no solo, a geração de gás, e o custo dos terrenos. A compostagem trabalha com determinados tipos de resíduos que pode coexistir com aplicações agrícolas, sem que isso represente a contaminação do solo [3]. A utilização de resíduos como combustível é limitada a demanda de energia elétrica e/ou sistema térmico [4]. Na medida em que existam diferentes usos para os resíduos, se aumentam as alternativas para aliviar o problema [5]. A sugestão oferecida aqui é o uso de resíduos orgânicos como matéria-prima para fabricação de ferro, por pirólise. As pelotas de auto-redutoras encaixam muito bem para esta finalidade. Elas consistem de uma mistura de carvão e minério de ferro com adição de pós fluxantes, pelotizadas com um aglomerante, que pode ser orgânico ou inorgânico. A vantagem em relação aos métodos tradicionais é a melhoria da cinética de reação entre a partícula de óxido de ferro e a de carvão [6]. No caso do carvão possuir um elevado teor de cinzas, isto pode ser reduzido por moagem seguido de um processo de flotação. As cinzas do processo podem ser utilizadas na construção [7].

\section{MATERIAIS E MÉTODOS}

O minério de ferro foi previamente secado e quarteado, seguindo a norma ASTM E 877-03, intitulada determinações para selecionar e preparar amostras de minério de ferro e outros materiais correlatos. Na tabela 1, mostra-se a composição química do minério de ferro utilizado nas experiências. Na figura 1, apresenta-se a distribuição do tamanho das partículas do minério de ferro hematítico.

Tabela 1. Composição química do minério de ferro hematítico (\% massa).

\begin{tabular}{|c|c|c|c|}
\hline $\mathrm{Fe}$ & 65,39 & $\mathrm{CaO}$ & 0,017 \\
\hline $\mathrm{FeO}$ & 0,22 & $\mathrm{MgO}$ & 0,046 \\
\hline $\mathrm{SiO}_{2}$ & 3,01 & $\mathrm{TiO}_{2}$ & 0,077 \\
\hline $\mathrm{Al}_{2} \mathrm{O}_{3}$ & 0,77 & $\mathrm{~K}_{2} \mathrm{O}$ & - \\
\hline
\end{tabular}

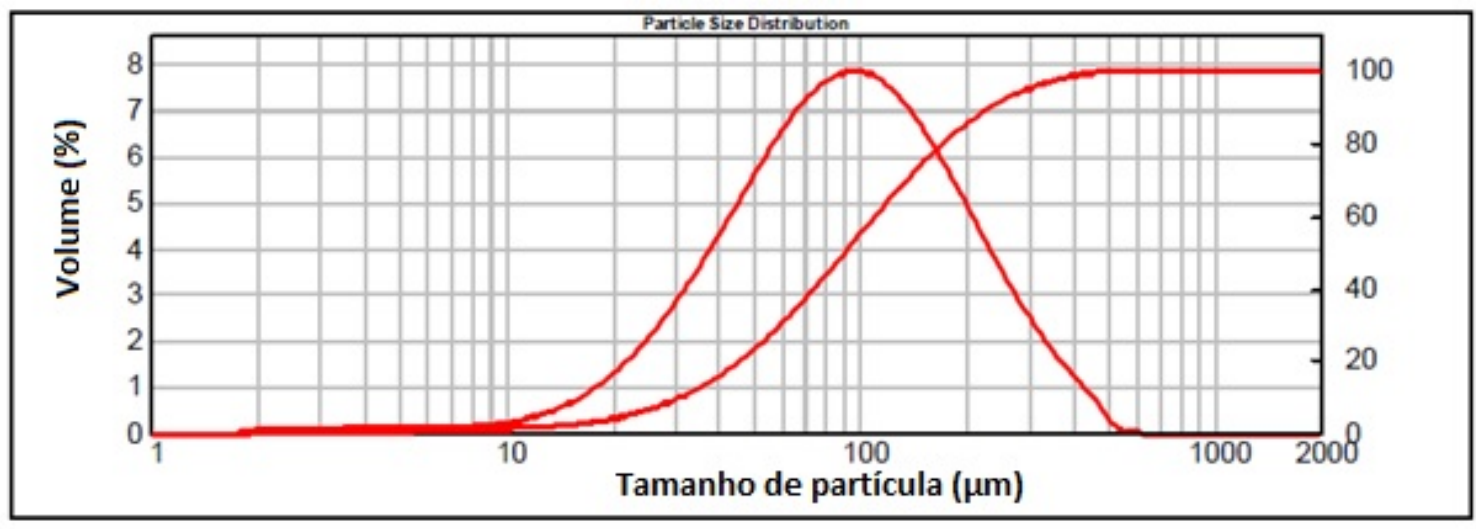

Figura 1. Distribuição do tamanho de particular do minério de ferro. 
Na figura 2 Tem-se a montagem experimental para a pirólise dos resíduos em ausência de oxigênio. Ela consiste de uma retorta em aço 306, aquecida em um forno de resistências de $8 \mathrm{KVA}$, Dentro da mesma são colocados os resíduos orgânicos previamente secados em uma estufa a $120^{\circ} \mathrm{C}$ durante 24 horas. $4 \mathrm{~kg}$ de material são aquecidos a $600^{\circ} \mathrm{C}$ por aproximadamente 3 Horas, Ate que a evolução dos voláteis diminui significativamente.

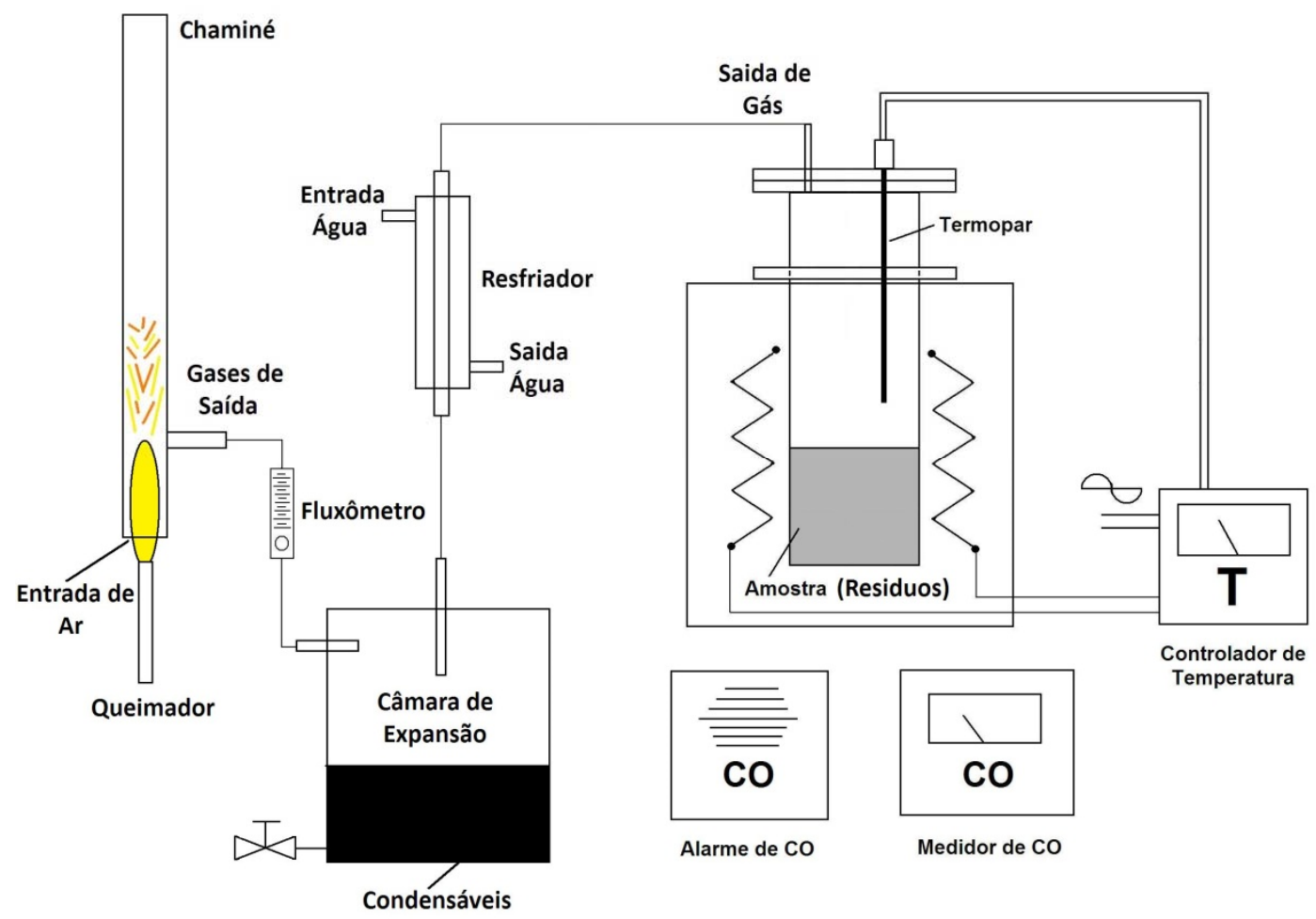

Figura 2. Montagem Experimental para a pirólise de resíduos.

Os voláteis São resfriados com ajuda de uma camisa de água, e posteriormente separados em uma câmara de expansão. $O$ volume dos gases de saída é medido com ajuda de um fluxômetro e posteriormente queimados. $O$ ar do laboratório é continuamente monitorado pela presença de gás CO nos gases de saída para reduzir perigos de explosão ou envenenamento. $O$ carvão que fica dentro da retorta posteriormente é pesado e moído.

$\mathrm{Na}$ Tabela 2 tem-se a composição aproximada dos resíduos. É importante ressaltar a dificuldade de padronizar os resíduos, pois vai depender da composição majoritária dele, do local e as condições onde ele é obtido. Por esse motivo, os dados aqui reportados são meramente ilustrativos, os quais estão sujeitos às mudanças em função da composição dos resíduos.

Tabela 2. Composição aproximada dos resíduos usados nesta experiência (\% massa).

\begin{tabular}{|c|c|c|}
\hline C fixo & Matéria volátil & $\mathrm{H}_{2} \mathrm{O}$ \\
\hline 35,5 & 43,2 & 21,3 \\
\hline
\end{tabular}

No processo de pirólise, os gases de saída possuem uma fase condensável (fase líquida) e uma fase não condensável (fase gasosa). A fase condensável esta composta por uma fração pesada (majoritariamente alcatrão), uma fração media de orgânicos leves e água. Os gases de saída estão compostos por uma mistura de $\mathrm{CO}, \mathrm{CO}_{2}$, Metano e hidrogênio. Na tabela 3 têm-se as porcentagens das frações 
obtidas neste trabalho. A fração pesada, o alcatrão, é utilizado na aglomeração das pelotas auto-redutoras.

Tabela 3. Composição aproximada da material volátil (\% massa).

\begin{tabular}{|c|c|c|}
\hline Fração Pesada & Fração Media & Fração Gasosa \\
\hline 18,5 & 28,3 & 53,2 \\
\hline
\end{tabular}

$\mathrm{Na}$ tabela 4 tem-se a análise imediata do carvão de resíduos a $900^{\circ} \mathrm{C}$ mostrando uma fração restante de matéria volátil e um baixo teor em cinzas, o qual é muito dependente do tipo de resíduos empregados. A cinza do carvão pode ser reduzida por trituração e flotação do carvão [8].

Tabela 4. Análise imediata do carvão de resíduos (\% massa).

\begin{tabular}{|c|c|c|}
\hline Carbono fixo & Matéria Volátil & Cinzas \\
\hline 90,7 & 6,1 & 3,2 \\
\hline
\end{tabular}

$\mathrm{Na}$ tabela 5 tem-se a composição aproximada das cinzas do carvão de resíduos. Estas cinzas podem ser empregadas na construção civil [7].

Tabela 5. Composição química das cinzas (\% massa)

\begin{tabular}{|c|c|c|}
\hline $\mathrm{SiO}_{2}$ & $\mathrm{Al}_{2} \mathrm{O}_{3}$ & $\mathrm{CaO}$ \\
\hline 67,3 & 25,4 & 7,3 \\
\hline
\end{tabular}

$\mathrm{Na}$ figura 3 temos a distribuição granulométrica do pó de Carvão utilizado na preparação das pelotas auto-redutoras.

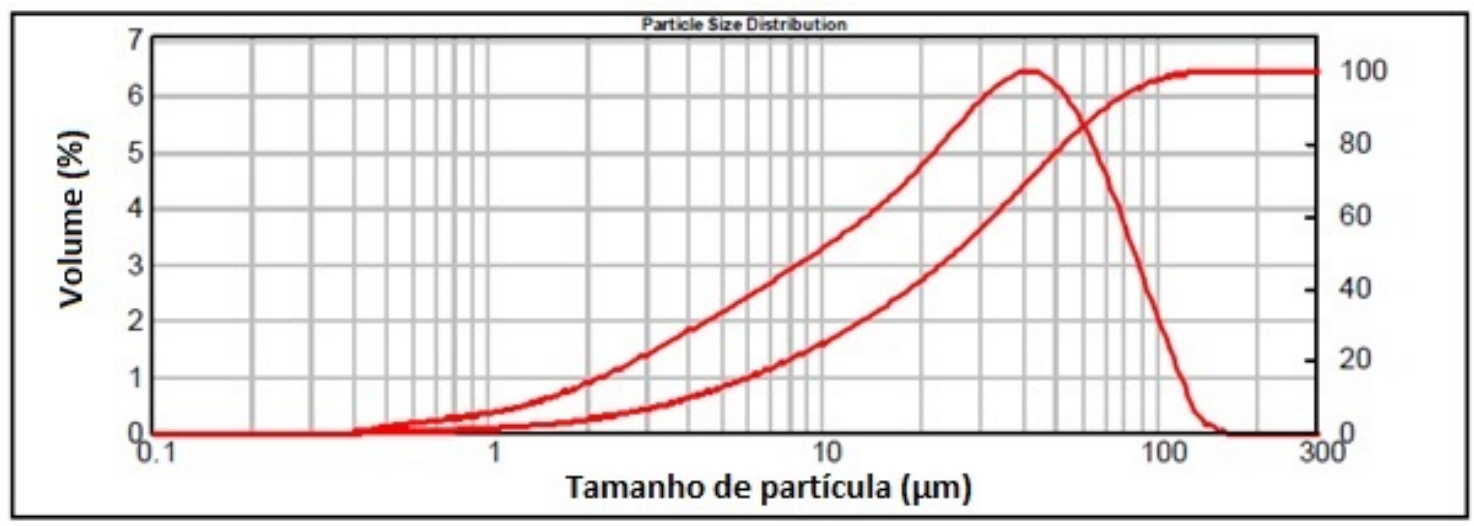

Figura 3. Distribuição do tamanho de partícula do carvão de resíduos após moagem.

Foram realizadas pelotas auto-redutoras com $79 \%$ de minério de ferro, $20 \%$ de carvão de resíduos, $1 \%$ de $\mathrm{CaO}$, Aglomerando com 3, 5 e 10\% de alcatrão para medir o efeito da concentração na resistência a compressão (ISO 4700), No teste de quedas e na resistência a abrasão a quente (ISO 3271), figura 4. 


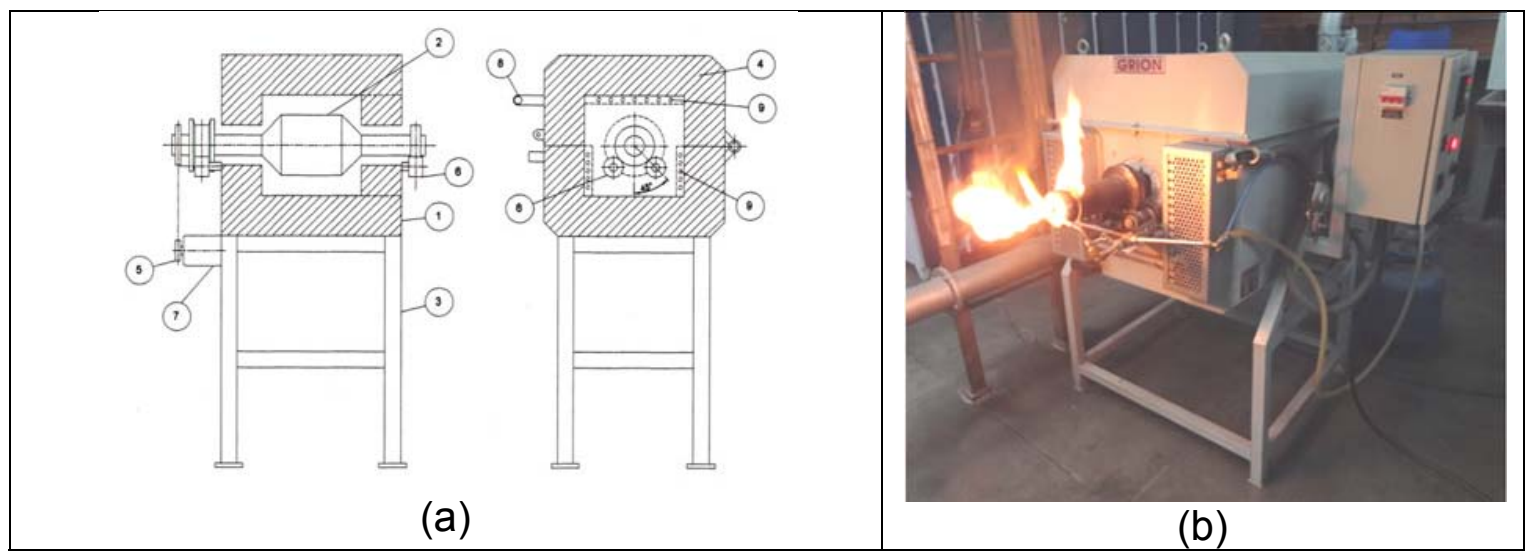

Figura 4. Forno rotativo para efetuar o teste de abrasão a quente (ISO 3271). a) Montagem. b) Em operação.

Foram elaboradas as curvas de perda de massa a 600,950 e $1200^{\circ} \mathrm{C}$. Com uma massa de $1,5 \mathrm{~kg}$ de pelotas, foi realizado um teste de fusão a $1600^{\circ} \mathrm{C}$ no forno de indução.

\section{RESULTADOS E DISCUSSÃO}

$\mathrm{Na}$ figura 5 tem-se a curva da resistência à compressão das pelotas auto-redutoras elaboradas com carvão de resíduos e aglomeradas com alcatrão, após ser processadas a $600^{\circ} \mathrm{C}$ durante 1 hora. A resistência à compressão aumenta com o incremento do ligante, sendo que para uma adição de $10 \%$ de alcatrão se atinge uma resistência media de $52 \mathrm{kgf}$ por pelota, resistência considerada excelente para uma pelota auto-redutora.

$\mathrm{Na}$ figura 6 temos o resultado do teste de quedas como uma função da porcentagem de alcatrão. O número de quedas aumenta com o aumento da concentração de alcatrão, resultando em um número de 400 quedas ate ruptura com $10 \%$ de alcatrão. A Norma estabelece um número mínimo de cinco quedas, mostrando um comportamento favorável.

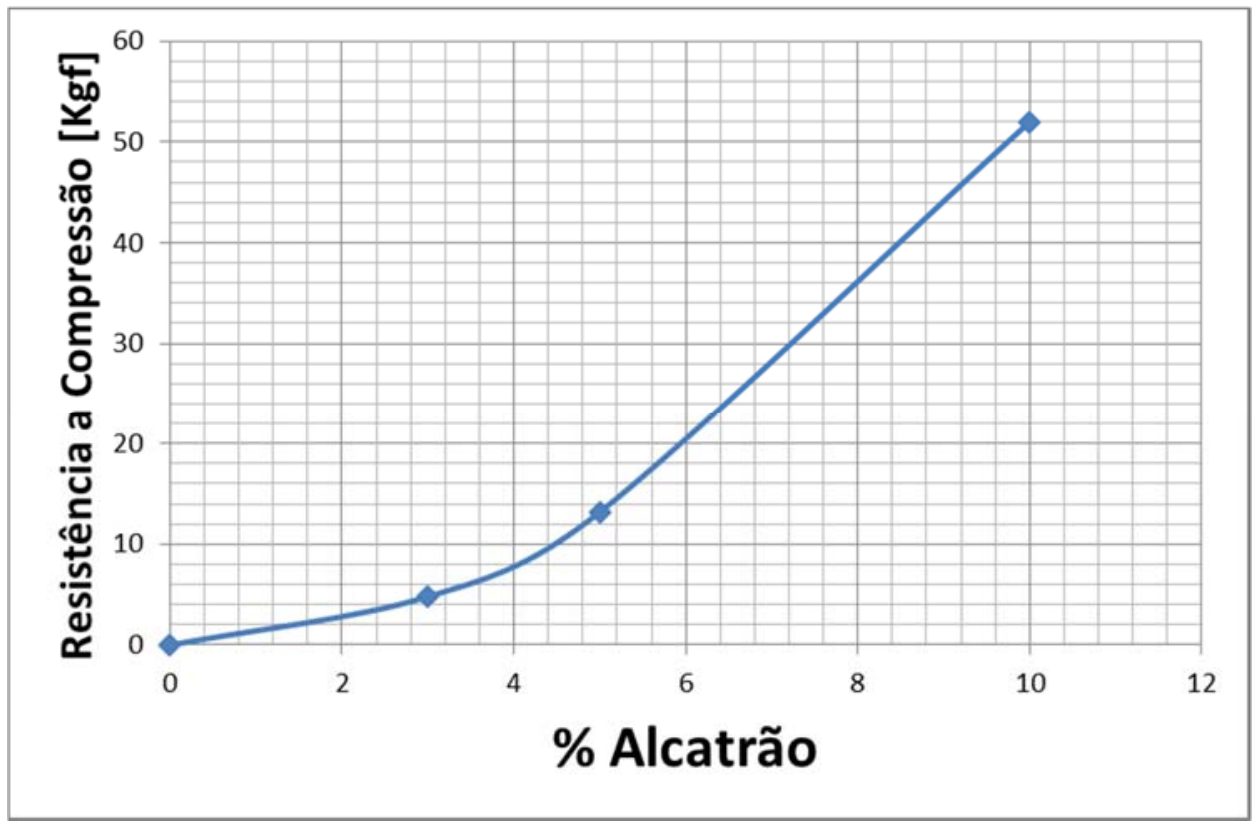

Figura 5. Resistência à compressão em função da concentração de alcatrão, após tratamento térmico a $600^{\circ} \mathrm{C}$ por 1 hora. 


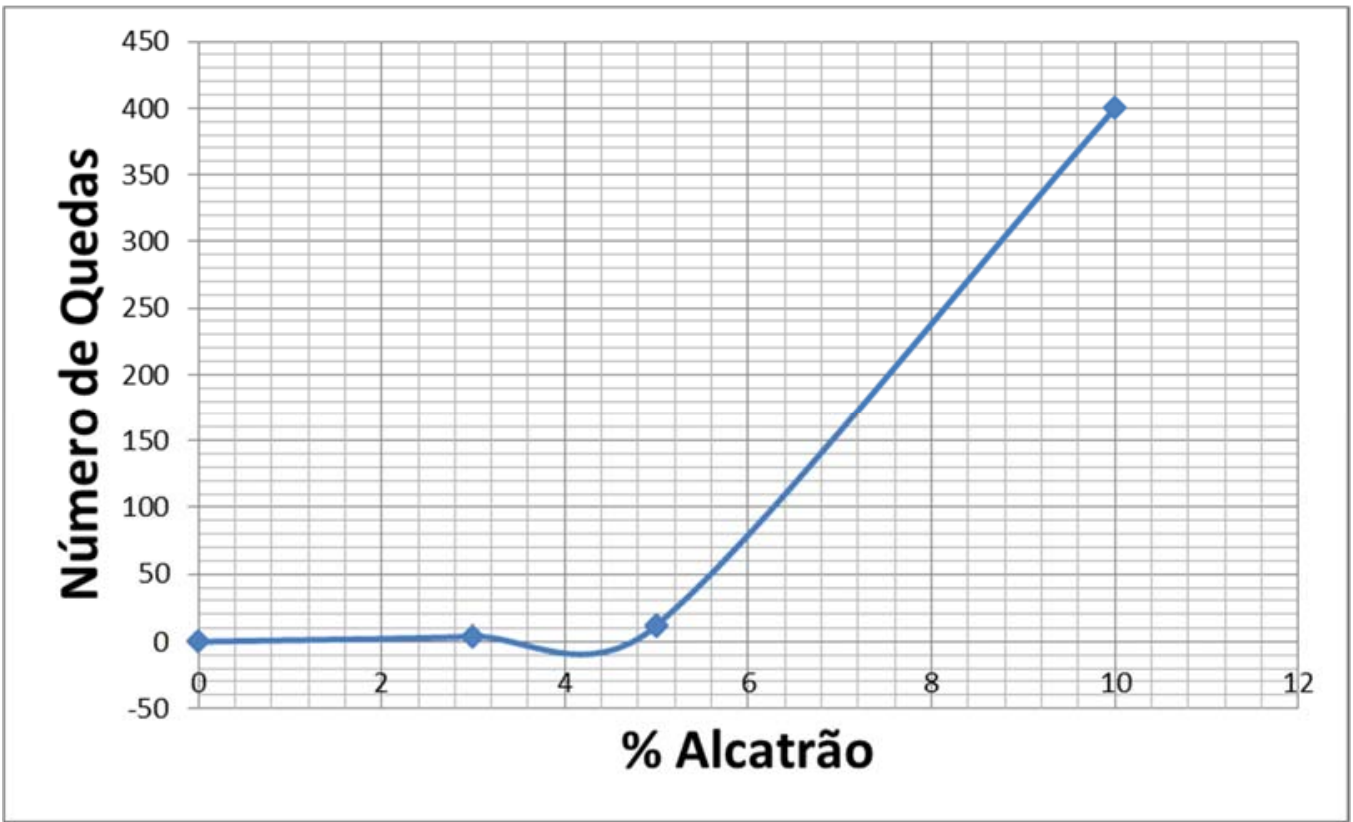

Figura 6. Número de quedas em função da concentração de alcatrão, após tratamento térmico a $600^{\circ} \mathrm{C}$ por 1 hora.

Na figura 7 tem-se uma imagem de microscopia MEV mostrando a formação de coque entre as partículas de minério de ferro e o carvão de resíduos, observando-se o bom grau de aglomeração obtida. As partículas de minério de ferro ficam presas em uma massa bastante uniforme de carvão coqueificado. Estas pelotas autoredutoras tem uma estabilidade térmica muito boa e uma menor quantidade de escória se comparados com outros aglomerantes como, por exemplo, o cimento ou bentonita.

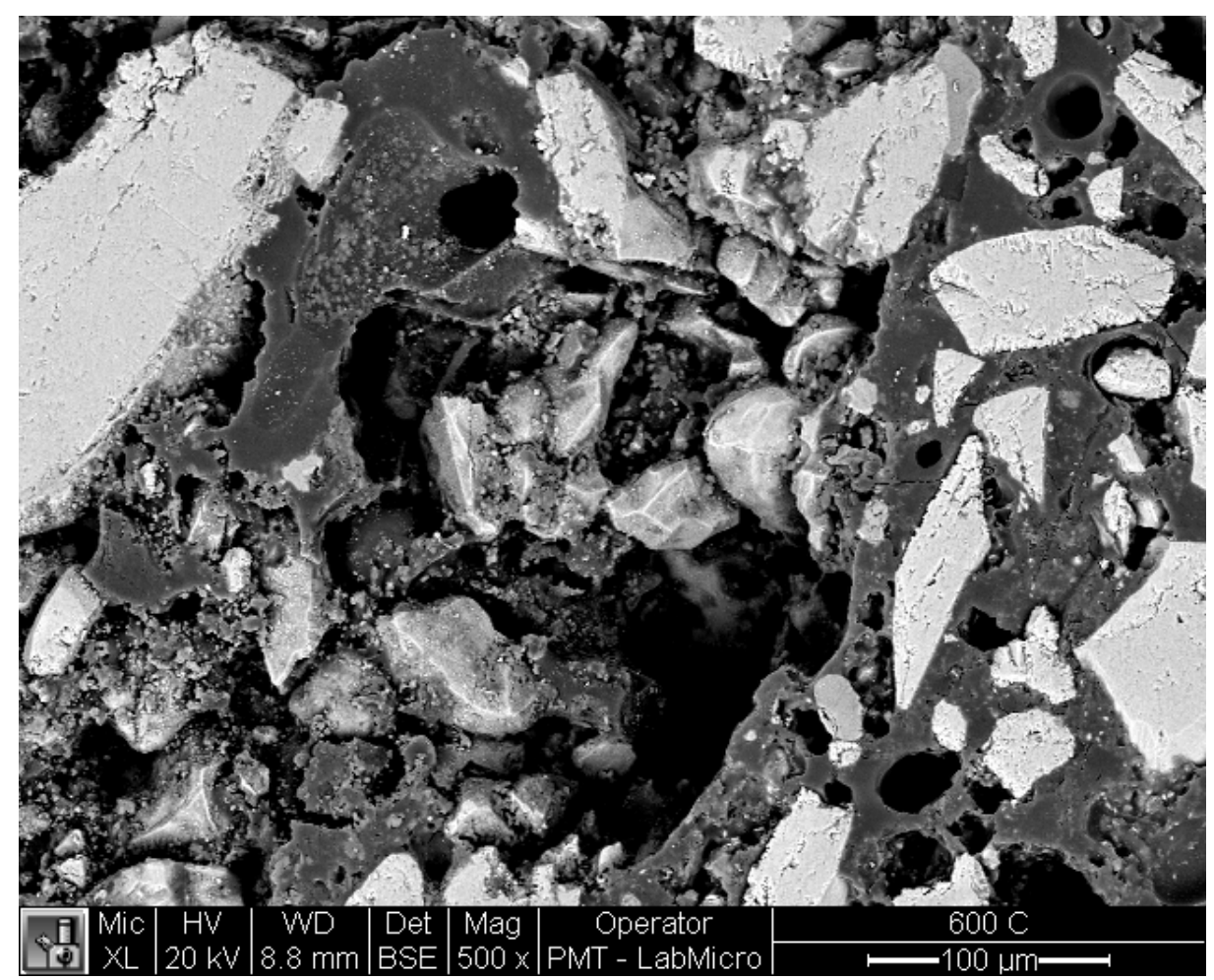

Figura 7. Imagem de microscopia eletrônica de varredura MEV de uma pelota auto-redutora aglomerada com $10 \%$ de alcatrão e processada a $600^{\circ} \mathrm{C}$ por uma hora. 
$\mathrm{Na}$ figura 8 temos o resultado do teste de abrasão quente, mostrando que 96,5\% estão acima de 6,33 milímetros (norma $\geq 90 \%$ ) e 3,2\% inferior a $-0,5 \mathrm{~mm}$ (Norma $\leq 5 \%$ ), o que representa um excelente desempenho na abrasão a quente.

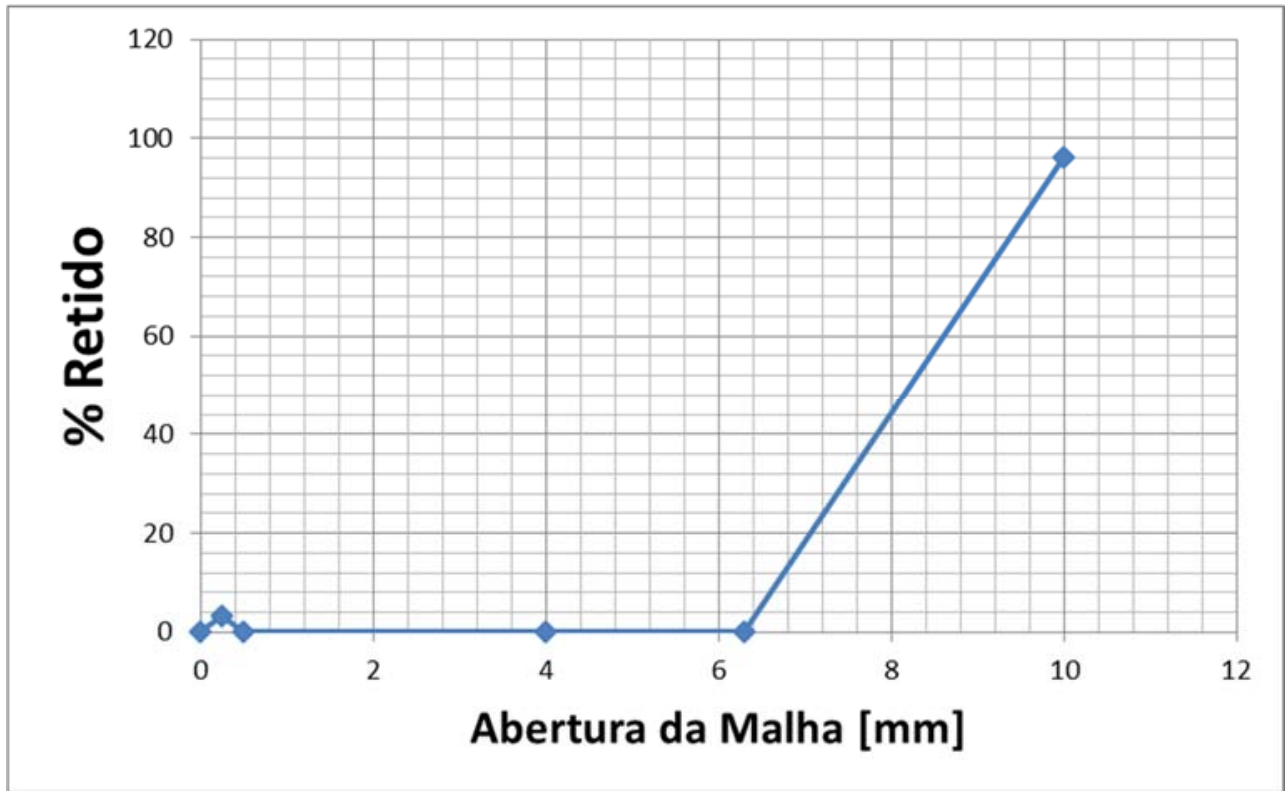

Figura 8. Distribuição do tamanho de partículas após teste de abrasão a quente a $950^{\circ} \mathrm{C}$ e 200 rev.

$\mathrm{Na}$ figura 9 temos as curvas de perdas de massa, para uma pelota auto-redutora seca, não processada termicamente. A uma temperatura de $600^{\circ} \mathrm{C}$ temos uma perda aproximada de $10 \%$ em massa no lapso de 1 hora. Esta perda corresponde à perda dos voláteis nesta faixa de temperatura. A $950^{\circ} \mathrm{C}$ tem-se uma perda de massa de aproximadamente $25 \%$ em 1 hora, esta perda corresponde a os voláteis e a uma parte da redução do minério de ferro. $\mathrm{A} 1200^{\circ} \mathrm{C}$ temos uma perda de massa de aproximadamente $42 \%$ em 1 hora que representam a eliminação total dos voláteis e a totalidade da redução.

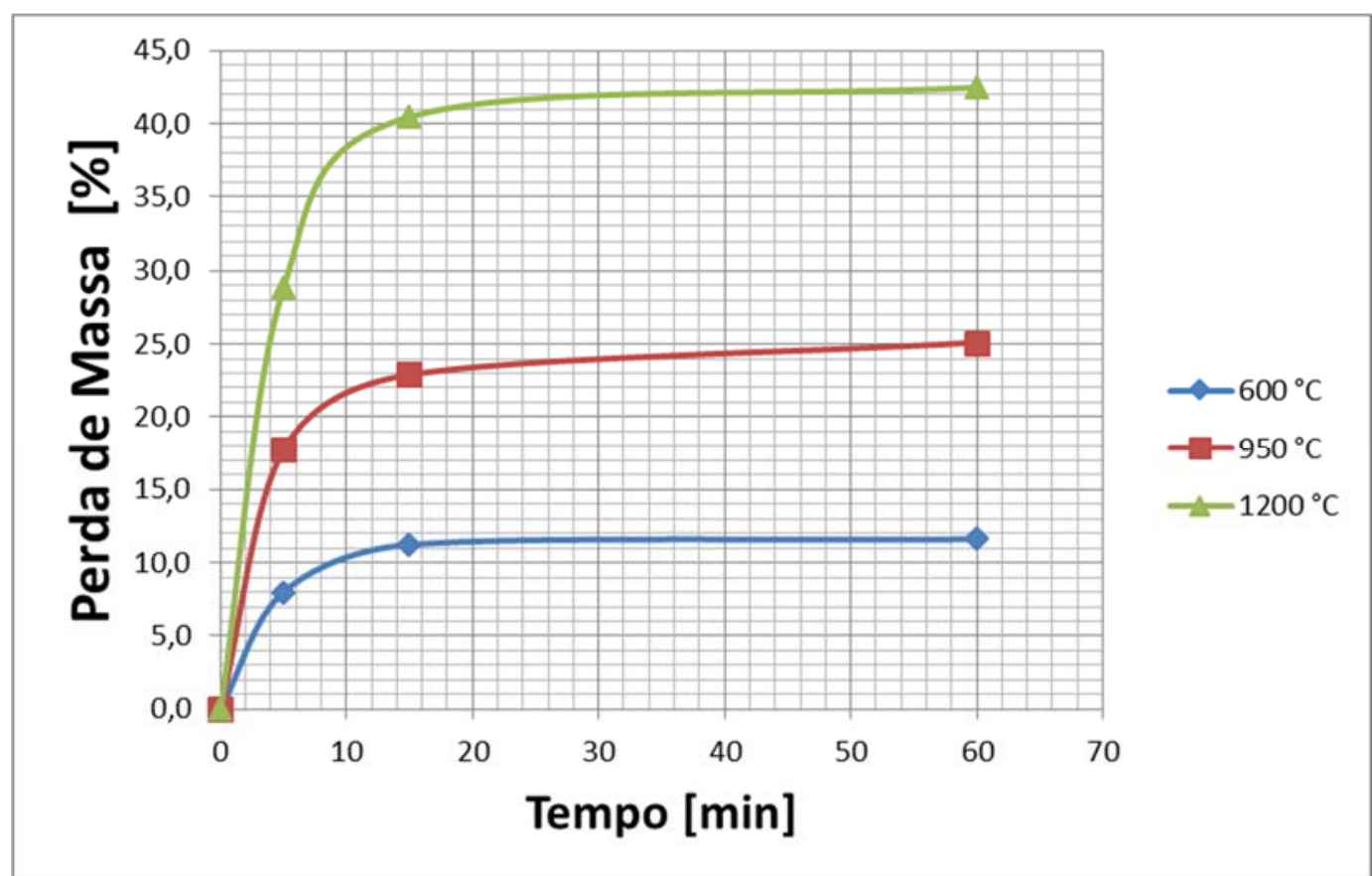

Figura 9. Perda de massa a partir de uma pelota sem processar, a 600,950 e $1200^{\circ} \mathrm{C}$. 
$\mathrm{Na}$ tabela 7 Tem-se a descrição do teste de fusão a $1600^{\circ} \mathrm{C}$ no forno de indução, com uma massa inicial de pelotas auto-redutoras de $1500 \mathrm{~g}$. Estas pelotas foram previamente processadas No forno de indução a $950^{\circ} \mathrm{C}$, obtendo-se a perda de massa nessa temperatura. Após a fusão, a massa final de ferro e escoria são obtidas. Os $6,4 \%$ da escoria final tem sua origem principalmente na ganga do minério e na adição de $\mathrm{CaO}$ como fluxante.

Tabela 7. Massa original das pelotas auto-redutoras, das pelotas processadas em forno rotativo a $950^{\circ} \mathrm{C}$, massa do ferro e da escória obtidas.

\begin{tabular}{|c|c|c|c|c|}
\hline $\mathrm{m}$ Inicial [g] & $\mathrm{m} 950{ }^{\circ} \mathrm{C}[\mathrm{g}]$ & $\mathrm{m} \mathrm{Fe}[\mathrm{g}]$ & $\mathrm{m}$ Escória [g] & \% Escória \\
\hline 1500,0 & 1123,9 & 704,8 & 45,2 & 6,4 \\
\hline
\end{tabular}

\section{CONCLUSÕES}

- E possível produzir carvão de resíduos orgânicos por pirólise em ausência de oxigênio.

- Os voláteis do processo servem como fonte de biocombustíveis.

- O Alcatrão do processo é um excelente ligante.

- É possível produzir ferro com carvão de resíduos com o uso de pelotas autoredutoras.

- As pelotas auto-redutoras podem ser um processo importante para o problema do lixo.

- Com uma adição de $10 \%$ de alcatrão após processamento a $600^{\circ} \mathrm{C}$ se consegue uma resistência à compressão de $52 \mathrm{Kgf}$. e uma resistência a quedas equivalente a 400 .

- Na medida em que aumenta a concentração de alcatrão na pelota, aumenta a resistência à compressão, a quedas e a abrasão a quente.

\section{REFERÊNCIAS}

1. Mc Leod, F; Cherrett, T; Quantifying the transport impacts of domestic waste collection strategies, Waste Management, n. 28, 2008, 2271-2278.

2. Yau, Y; Domestic waste recycling, collective action and economic incentive: The case in Hong kong, Waste Management, n. 30, 2010, 2440-2447.

3. Hudson, A; White, J; Beaven, R; Powrie, W; Modelling the compression Behaviour of landfilled domestic waste, Waste Management, n.24, 2004, 259-269.

4. Bruckner, A; Schafers, H; Peters, I; Lavemann, E; Using industrial and commercial waste heat for residential heat supply: A case study From Hamburg, Germany, Sustainable cities and society, n. 13, 2014, 139-142.

5. $\mathrm{Li}, \mathrm{J}$; The key to avoiding secondary pollutants in the incineration of domestic waste lies in prevention, the The 7th International Conference on Waste Management and Technology, Procedia Environmental Sciences, n. 16, 2012, 669-673.

6. Nogueira, A. Mourão, M; Takano, C; Santos, D; Effect of slag composition on iron nuggets formation from carbon composite pellets. . Materials Research, v.13, 2010, 191195.

7. Sobiecka, E; Obraniak, A; Antizar-Ladislao, B. Influence of mixture ratio and $\mathrm{pH}$ to solidification/stabilization process of hospital solid waste incineration ash in Portland cement, Chemosphere n.111, 2014, 18-23.

8. Wang, B; Peng, Y; The interaction of clay minerals and saline water in coarse coal flotation, Fuel, n. 134, 2014, 326-332. 